\title{
First-principles stabilization of an unconventional collinear magnetic ordering in distorted manganites
}

\author{
S. Picozzi \\ CNR-INFM, CASTI Regional Lab, I-67010 Coppito (L'Aquila), Italy
}

K. Yamauchi

ISIR-SANKEN, Osaka University, Mihogaoka 8-1, Ibaraki, Osaka 567-0047, Japan

G. Bihlmayer and S. Blügel

Institut für Festkörperforschung, Forschungszentrum Jülich, 52425 Jülich, Germany

(Received 12 April 2006; revised manuscript received 11 July 2006; published 1 September 2006)

\begin{abstract}
First-principles calculations have been performed for different collinear magnetic orderings in orthorhombic manganites, such as $\mathrm{HoMnO}_{3}, \mathrm{TbMnO}_{3}$, and $\mathrm{YMnO}_{3}$, showing large $\mathrm{GdFeO}_{3}$-like distortions. Our results suggest that the AFM- $E$ type ordering, experimentally observed in $\mathrm{HoMnO}_{3}$ and recently proposed from model Hamiltonian studies, is indeed the magnetic ground state. Its stability is strongly connected with octahedral distortions and points to the relevance of structural more than chemical effects. The calculated exchange constants, extracted from a Heisenberg model used to fit the first-principles total energies, show that the ferromagnetic in-plane nearest-neighbor coupling is reduced compared to less-distorted manganites, such as $\mathrm{LaMnO}_{3}$. In parallel, the antiferromagnetic next-nearest-neighbor coupling along planar Mn-O-O-Mn paths in highly distorted manganites plays a relevant role in the stabilization of the AFM- $E$ spin configuration. In agreement with experiments, the density of states shows that this phase is insulating with an indirect band gap of $\sim 0.5 \mathrm{eV}$.
\end{abstract}

DOI: 10.1103/PhysRevB.74.094402

PACS number(s): 75.47.Lx, 75.30.Et, 71.70.--d, 71.15.Mb

\section{INTRODUCTION}

Rare-earth manganites show a fascinating variety of physical phenomena, ${ }^{1,2}$ ranging from their unusual magnetotransport properties (leading to the well known colossal magnetoresistance ${ }^{2}$ ) to peculiar charge, spin, and orbital orderings to recently discovered ferroelectricity. ${ }^{3,4}$ It is widely accepted that the intriguing manganites physics is intimately related to the profound interplay between the crystal lattice and the spin, charge, and orbital degrees of freedom. In $R \mathrm{MnO}_{3}$ with light rare-earth $(R)$ cations $(R=\mathrm{La}, \mathrm{Pr}, \mathrm{Nd})$, the collinear antiferromagnetic $A$-type (AFM- $A$ ) ordering [i.e., ferromagnetically (FM) coupled ac layers antiferromagnetically aligned along the $b$ direction in the Pnma setting] is favored, whereas manganites toward the end of the series $(R=\mathrm{Tb}, \mathrm{Dy}, \mathrm{Y})$ exhibit a sinusoidal magnetic structure defined by the propagation vector $\left(k_{x}, 0,0\right)$. Recently, an AFM phase has been observed in late- $R \mathrm{HoMnO}_{3}$ (Ref. 5) as well as in nickelates, ${ }^{6}$ with an "up-up-down-down" spin ordering in the $\mathrm{MnO}_{2}$ planes (the so-called " $E$-type"in the WollanKoehler notation ${ }^{7}$ ), i.e., two Mn with up spins are alternated by two Mn with down spins along the principal axes of the in-plane cubic unit cell, whereas the spins are reversed from plane to plane in the perpendicular direction. It is surprising that only very recently this phase has been proposed in undoped manganites, ${ }^{8,9}$ considered well-understood systems, where the $E$-type ordering was found to be stabilized in a wide region of the phase space.

The focus of the present work is therefore to compare our results with experimental observations suggesting AFM- $E$ as the magnetic ground state in $\mathrm{HoMnO}_{3}$ and, more in general, to deeply investigate this nonconventional magnetic order- ing, focusing in particular on the link between magnetic and structural properties. In closer detail, we consider different collinear magnetic alignments for three highly distorted manganites, $\mathrm{HoMnO}_{3}, \mathrm{TbMnO}_{3}$, and $\mathrm{YMnO}_{3}$, in order to find the stable magnetic configuration and compare our results for the most studied $\mathrm{LaMnO}_{3}$. Surprisingly, we find that the AFM-E is the ground-state collinear phase for $\mathrm{HoMnO}_{3}, \mathrm{TbMnO}_{3}$, and $\mathrm{YMnO}_{3}$ and that its stabilization is mainly related to octahedral distortions that decrease the in-plane FM interaction between $e_{g}$ states.

\section{STRUCTURAL AND COMPUTATIONAL DETAILS}

The simulations have been performed within the generalized gradient approximation (GGA) to the exchangecorrelation potential in the density-functional framework. The all-electron full-potential linearized augmented planewave $(\text { FLAPW })^{10}$ formalism in the FLEUR ${ }^{11}$ implementation has been used. Muffin-tin radii have been set to 2.5, 2.5, 2.5, 2.5, 2.0, and 1.5 a.u. for $\mathrm{Tb}, \mathrm{Y}, \mathrm{La}, \mathrm{Ho}, \mathrm{Mn}$, and $\mathrm{O}$ atoms, respectively, whereas the wave-function cutoff was chosen as 3.8 a.u. ${ }^{-1}$. Convergency was carried out using 24 special $\mathbf{k}$ points in the orthorhombic Brillouin zone, whereas finer quantities (such as differences in the total energy) were checked using 192 special $\mathbf{k}$ points.

In order to accurately treat within density functional theory the $4 f$ electrons, which typically occupy an unphysical position slightly above the Fermi level $\left(E_{F}\right)$ when considered as valence states, the Ho and $\mathrm{Tb} 4 f$ electrons were kept in the core as an open shell ${ }^{12}$ [with 7 and 1 (3) electrons in the up and down spin channel, respectively, for $\mathrm{Tb}(\mathrm{Ho})]$. An alternative solution to remove spurious effects related to 
(a)

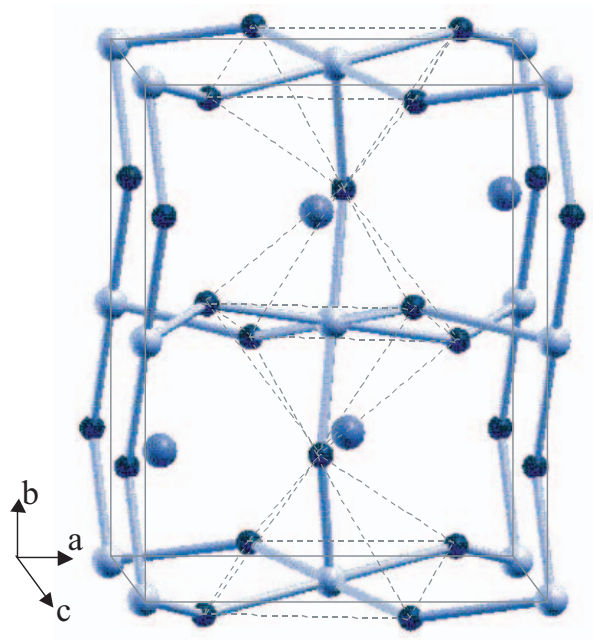

(b)

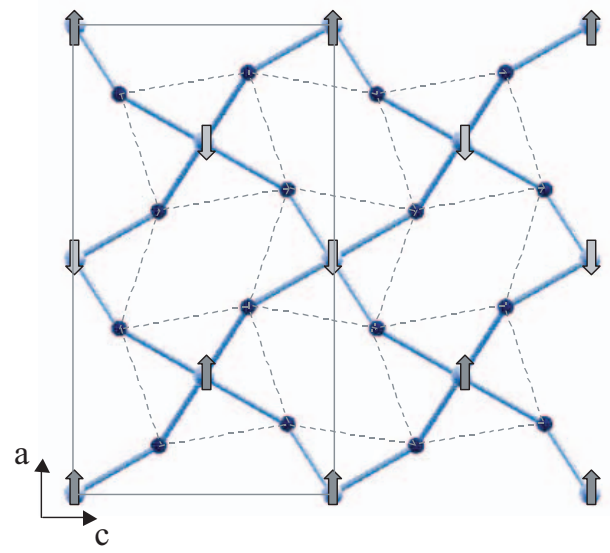

FIG. 1. (Color online) (a) The Pnma orthorhombic cell of $R \mathrm{MnO}_{3}$. Black, white, and gray spheres represent $\mathrm{O}, \mathrm{Mn}$, and $\mathrm{Tb}$ (or $\mathrm{Y}$ or La or Ho) atoms, respectively. (b) $a c$ in-plane view of $\mathrm{MnO}_{2}$ planes, showing the large octahedral distortions and "up-updown-down" zigzag spin ordering. Solid lines mark the in-plane projected unit cell. the wrong energy position of $\mathrm{Tb} 4 f$ electrons is to treat them as valence states using an LSDA $+U$ approach. ${ }^{13}$ Tests performed on the undistorted perovskite-like cubic phase using LSDA $+U$ (Ref. 14) on the $4 f$ states showed that the resulting electronic structure is unchanged with respect to the " $4 f$-in-core" treatment.

The unit cell in the $\mathrm{GdFeO}_{3}$-like orthorhombic phase shows the Pnma symmetry (20 atoms/unit cell, choosing $b$ as the longest axis), with enormous distortions with respect to the ideal cubic perovskite (see Fig. 1): due to the Jahn-Teller instability shown by the $\mathrm{Mn}^{3+}$ ion, with electronic configuration $d^{4}\left(t_{2 g}^{3} e_{g}^{1}\right)$, oxygen octahedrons are highly distorted and tilted (the average $\mathrm{Mn}-\mathrm{O}-\mathrm{Mn}$ angle is $\sim 145^{\circ}, \sim 142^{\circ}$, and $\sim 144^{\circ}$ for $\mathrm{TbMnO}_{3}, \mathrm{HoMnO}_{3}$, and $\mathrm{YMnO}_{3}$, respectively, to be compared with the much larger value of $\sim 155^{\circ}$ in $\mathrm{LaMnO}_{3}$ ). We recall that for $\mathrm{YMnO}_{3}$ (and for other $R$ smaller than $\mathrm{Tb}$ ), the orthorhombic perovskite structure is no longer stable, the hexagonal nonperovskite structure competing in stability. However, the transition to the metastable orthorhombic Pnma can be obtained by high-pressure synthesis. ${ }^{15}$ The experimental lattice constants and internal positions according to neutron-diffraction data-very similar for the three compounds $\mathrm{HoMnO}_{3}, \mathrm{YMnO}_{3}$, and $\mathrm{TbMnO}_{3}$ - have been used throughout the work. ${ }^{15}$ In order to separate chemical and structural effects, we have also considered (a) $\mathrm{LaMnO}_{3}$, having less distorted octahedrons and (b) two "artificial" systems, namely (i) $\mathrm{YMnO}_{3}$ in the $\mathrm{LaMnO}_{3}$ structure and (ii) $\mathrm{LaMnO}_{3}$ in the $\mathrm{YMnO}_{3}$ structure (denoted in the following as $\left[\mathrm{YMnO}_{3}\right]_{\mathrm{La}}$ and $\left[\mathrm{LaMnO}_{3}\right]_{\mathrm{Y}}$, respectively).

\section{RESULTS AND DISCUSSION}

Let us first focus on $\mathrm{YMnO}_{3}, \mathrm{TbMnO}_{3}, \mathrm{HoMnO}_{3}$, and $\mathrm{LaMnO}_{3}$ in their equilibrium structure. In Table I, we report the total energies (calculated per formula unit containing one $\mathrm{Mn}$ atom) with respect to the FM phase. If we restrict to the FM and AFM- $A, C$, and $G$ orderings (i.e., we neglect possible next-nearest-neighbors AFM coupling), the situation for $\mathrm{HoMnO}_{3}, \mathrm{TbMnO}_{3}$, and $\mathrm{YMnO}_{3}$ is common to the well studied $\mathrm{LaMnO}_{3}$, where the most stable magnetic configuration, as experimentally observed ${ }^{16}$ and theoretically confirmed, ${ }^{17,18}$ is the AFM- $A$ type.${ }^{19}$ However, the inclusion of the AFM- $E$ in the subset of considered magnetic configurations largely changes the scenario: interestingly, among the collinear magnetic orderings, (i) $\mathrm{HoMnO}_{3}$ show AFM- $E$ as a magnetic ground state, in excellent agreement with experiments, (ii) both $\mathrm{YMnO}_{3}$ and $\mathrm{TbMnO}_{3}$ show the AFM- $E$ type

TABLE I. Calculated total energy difference (in meV/Mn) with respect to the FM phase. Recall that (i) type- $E$ consists of intraplane up-up-down-down and interplane AFM coupling; (ii) type- $E^{*}$ consists of intraplane up-up-down-down and interplane FM coupling; (iii) type- $A$ consists of interplane AFM and intraplane FM coupling; (iv) type- $C$ consists of intraplane AFM and interplane FM coupling; (v) type- $G$ consists of both interplane and intraplane AFM coupling. Numbers in bold denote the ground state.

\begin{tabular}{lccccccc}
\hline \hline & $\mathrm{YMnO}_{3}$ & $\mathrm{HoMnO}_{3}$ & $\mathrm{TbMnO}_{3}^{(7 \uparrow, 1 \downarrow)}$ & $\mathrm{TbMnO}_{3}^{(4 \uparrow, 4 \downarrow)}$ & $\mathrm{LaMnO}_{3}$ & {$\left[\mathrm{YMnO}_{3}\right]_{\mathrm{La}}$} & {$\left[\mathrm{LaMnO}_{3}\right]_{\mathrm{Y}}$} \\
\hline $\mathrm{FM}$ & 0 & 0 & 0 & 0 & 0 & 0 & 0 \\
AFM- $E$ & $-\mathbf{4 4}$ & $-\mathbf{3 7}$ & $-\mathbf{3 2}$ & $-\mathbf{4 5}$ & -2 & +13 & $\mathbf{- 6 1}$ \\
AFM- $E^{*}$ & -23 & -12 & -11 & -30 & +2 & +15 & -34 \\
AFM- $A$ & -29 & -16 & -8 & -21 & $-\mathbf{1 7}$ & $-\mathbf{7}$ & -38 \\
AFM- $C$ & -10 & -2 & +17 & +9 & +58 & +78 & -33 \\
AFM- $G$ & -24 & -14 & +4 & -17 & +64 & +88 & -52 \\
\hline \hline
\end{tabular}


as stable configuration, and (iii) in $\mathrm{LaMnO}_{3}$ the AFM- $E$ is also not too high in energy compared to the AFM- $A$ stable configuration. Indeed, this confirms what was proposed via model Hamiltonians, i.e., the occurrence of the AFM-E phase adjacent to the AFM- $A$ in parameter space and competing with the FM metallic phase as well. ${ }^{8}$ The differences between $\mathrm{HoMnO}_{3}, \mathrm{TbMnO}_{3}$, and $\mathrm{YMnO}_{3}$-having very similar structural parameters-suggests that, to some extent, the $4 f$ moment affects the Mn ordering. In order to further highlight the effect of the spin polarization due to the $4 f \mathrm{~Tb}$ electrons, we considered a configuration in which these electrons are kept in the core, but they are equally shared between the majority and minority spins [4 in the up and 4 in the down channels, denoted as $(4 \uparrow, 4 \downarrow)]$, instead of the "normal" configuration-considered so far-in which there are 7 and 1 electrons in the up and down spin channels, respectively, denoted as $(7 \uparrow, 1 \downarrow)$. The results show that the energy ordering of the different phases in $\mathrm{TbMnO}_{3}^{(4 \uparrow, 4 \downarrow)}$ is very similar to $\mathrm{YMnO}_{3}$, therefore suggesting that the Mn-Mn coupling is appreciably affected by $\mathrm{Tb} 4 f$ electrons. In particular, due to the geometric location of $\mathrm{Tb}$ atoms (i.e., in between $a c$ planes), the main differences induced by the treatment of $\mathrm{Tb}$ $4 f$ states occur in the out-of-plane interactions, appreciably affected by the $4 f$ "spin-polarized" or "paramagnetic" electronic cloud in the $(7 \uparrow, 1 \downarrow)$ and $(4 \uparrow, 4 \downarrow)$ case, respectively.

At this point, one might infer that the stability of the AFM- $E$ is closely related to distortions (larger in $\mathrm{HoMnO}_{3}$, $\mathrm{YMnO}_{3}$, and $\mathrm{TbMnO}_{3}$ compared to $\mathrm{LaMnO}_{3}$ ). Indeed, this is confirmed by our "artificial" structures: in $\left[\mathrm{YMnO}_{3}\right]_{\mathrm{La}}$ the reduced distortions - compared to $\mathrm{YMnO}_{3}$ in equilibriumrestore the stability of the AFM-A (similar to $\mathrm{LaMnO}_{3}$ ), whereas the increased distortions in $\left[\mathrm{LaMnO}_{3}\right]_{\mathrm{Y}}$-compared to $\mathrm{LaMnO}_{3}$ at equilibrium-make the AFM- $E$ the ground state. Therefore, the stability of the AFM- $E$ seems to be mainly connected with structural (i.e., octahedral distortions) more than with chemical (i.e., identity of the rare-earth element) effects.

In order to investigate the role of electronic correlation on the stability of the different magnetic states, we performed some calculations for $\mathrm{YMnO}_{3}$ in the FM, AFM- $A$, and AFM$E$, according to an $\mathrm{LSDA}+U$ (or GGA $+U$ ) approach in the "atomic-limit"13 and varying the $U$ value from 0 to $8 \mathrm{eV}$, keeping the $U / J$ ratio fixed and choosing $J=0.15 U$. In Fig. 2(a), we show the difference between total energies in the AFM- $A$ and FM as well as in the AFM- $E$ and FM. For a complete description of the electronic and magnetic structure, we also report the variation of the magnetic moment and of the band gap as a function of the Coulomb parameter [see Figs. 2(b) and 2(c), respectively]. The band gap and the magnetic moment both increase as a function of $U$, with a strong and rather weak dependence on the Coulomb parameter, respectively. This is expected, since LSDA $+U$ tends to enhance the "localization" of Mn $d$ states (thereby increasing the magnetic moment) and to push unoccupied states up in energy (thereby increasing the band gap). As far as the magnetic ground state is concerned, our results show that the stability of the AFM- $E$ and AFM- $A$ is strongly affected by correlation effects: for values of $U$ larger than $4(7.5) \mathrm{eV}$, the FM is stabilized with respect to the AFM- $E$ (AFM- $A$ ). We

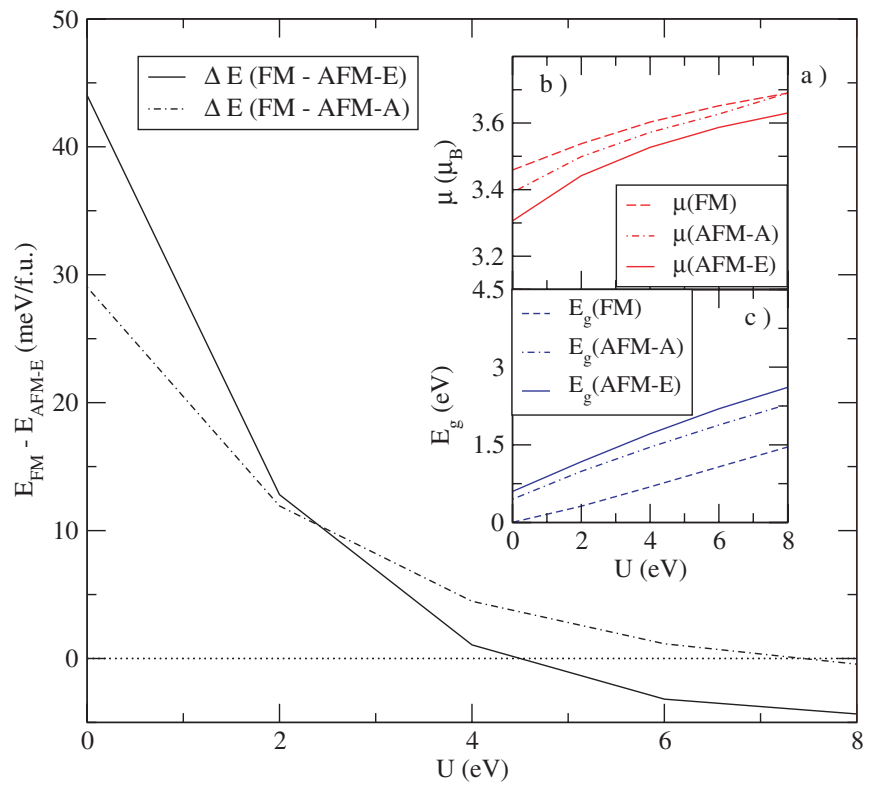

FIG. 2. (Color online) (a) Difference (in meV/formula unit) between the FM and AFM-E (black solid line) and the FM and AFM$A$ (black dot-dashed line) as a function of the Coulomb parameter for $\mathrm{YMnO}_{3} . J$ is set to $0.15 U$. The changes of (b) Mn magnetic moment and (c) band gap as a function of $U$ are also shown for the different phases: FM (dashed line), AFM- $A$ (dot-dashed line), and AFM-E (solid line).

remark that the LSDA $+U$ formalism might overestimate the tendency toward ferromagnetism, as already noted by Terakura and co-workers for $\mathrm{LaMnO}_{3}$ (Ref. 21) and consistently with our results in $\mathrm{YMnO}_{3}$. In addition, to our knowledge and at present, there are no spectroscopic works for $\mathrm{YMnO}_{3}$ in the orthorhombic phase, on the basis of which one could extract the value of the Coulomb parameter. So, the LSDA $+U$ results are mainly shown to warn the reader that the magnetic ground state may be affected by correlation effects. We hope our work will stimulate further experimental works on distorted manganites to gain additional insights into the accuracy of bare GGA versus GGA $+U$ in treating these systems.

More information on the magnetic interactions between $\mathrm{Mn}$ atoms can be gained by considering a Heisenberg Hamiltonian $^{20}$ and using this model to fit our GGAcalculated total energies. In particular, we consider the firstnearest-neighbor $\left(J_{\|}^{\mathrm{nn}}\right)$ and second-nearest-neighbor coupling $\left(J_{\|}^{\mathrm{nnn}}\right)$ in the $a c$ plane, as well as the first-and second-nearestneighbor coupling out of plane $\left(J_{\perp}^{1}\right.$ and $\left.J_{\perp}^{2}\right)$. Before discussing quantitatively our results, let us first recall what happens in the well-understood $\mathrm{LaMnO}_{3}$, showing a smaller $\mathrm{GdFeO}_{3}$-type distortion: the nn FM superexchange (SE) between $e_{g}$ spins competes with the AFM SE between $t_{2 g}$ spins. ${ }^{18}$ The delicate balance of these two mechanisms depends crucially on the $\mathrm{MnO}_{6}$ tilting and Jahn-Teller distortions. ${ }^{15}$ Indeed, this is evidenced by our results: for the heavily distorted compounds, the FM nn exchange parameters $\left(J_{\|}^{\mathrm{nn}} \sim-1\right.$ to $\left.2 \mathrm{meV}\right)$ are smaller in module ${ }^{9}$ than the much larger value in $\mathrm{LaMnO}_{3}\left(J_{\|}^{\mathrm{nn}} \sim-9 \mathrm{meV}\right)$. This can be 
explained considering that, upon large tilting (i.e., in $\mathrm{HoMnO}_{3}, \mathrm{TbMnO}_{3}$, and $\mathrm{YMnO}_{3}$ ), the overlap between $\mathrm{Mn}$ $3 d$ and $\mathrm{O} 2 p$ orbitals is not large enough to provide a strongly FM SE between Mn cations in the $a c$ plane. In turn, this alters the delicate balance with the nnn SE interactions through Mn-O-O-Mn paths along the $a$ direction. Upon significant $\mathrm{GdFeO}_{3}$ distortions, the large $J_{\|}^{\mathrm{nnn}}$ (of the order of few $\mathrm{meV}$ in all compounds) AFM coupling prevails, therefore stabilizing the AFM- $E$ ordering. Furthermore, the outof-plane coupling between $t_{2 g}$ spins $\left(J_{\perp}^{1}\right.$ of the order of a few $\mathrm{meV}$ ) keeps a strong AFM character along the $R$ series, whereas we obtain very small $(<0.5 \mathrm{meV}) J_{\perp}^{2}$ coupling perpendicular out of plane. Finally, the octahedral distortions also affect this interplanar coupling $\left(J_{\perp}^{1}\right.$ is smaller in $\mathrm{LaMnO}_{3}$ than in distorted manganites), therefore weakening the assumption, very common in model Hamiltonians, of restricting the study to a 2D square lattice, ${ }^{8,9}$ neglecting outof-plane effects.

Let us now discuss the electronic and magnetic properties of the AFM- $E$ phase, in terms of magnetic moments, band structures, and density of states. The GGA Mn local magnetic moment $\left(\sim 3.3 \mu_{B}\right)$ is reduced with respect to the Hund's rule value $\left(4 \mu_{B}\right)$, reflecting the hybridization with $\mathrm{O}$ atoms (slightly ferromagnetically polarized). The GGAcalculated total density of states and band structure for the AFM- $E \mathrm{YMnO}_{3}$ are reported in Fig. 3. Common to the well studied $\mathrm{LaMnO}_{3}$ and shown by the density of states projected on the muffin-tin spheres (not reported), there is a strong hybridization between $\mathrm{Mn} d$ and $\mathrm{O} p$ states. In particular, the bands lying just below $E_{F}$ [see highlighted states in Figs. 3(a) and 3(b)] and separated by the rest of the valence band are $\mathrm{Mn}-\mathrm{O}$ bands (occupied hybridized $e_{g}$-like states). In agreement with the experimentally observed insulating character, ${ }^{9}$ the resulting band structure shows an indirect band gap, with the conduction-band minimum at $\Gamma$ and the valence-band maximum at the Brillouin-zone edge along the [010]- $\Gamma$ line and-more or less degenerately—along the $[110]-\Gamma$ line. It is interesting to note that, even without the introduction of the on-site Coulomb $U$ repulsion, the AFM- $E$ configuration shows an energy gap, in agreement with the experimental observation of an insulating behavior. When comparing with the metallic character of the cubic perovskite-like systems (not shown), despite their FM or AFM spin alignments, the effect of the Jahn-Teller distortion and of the octahedral tilting is seen to result in a removal of states at the Fermi level by opening band gaps. ${ }^{17}$ As shown in Fig. 2(c), the precise value of the band gap is strongly affected by the inclusion of correlation effects: for the extreme case of $U=8 \mathrm{eV}$, the gap increases up to $\sim 2.5 \mathrm{eV}$. Finally, in order to have some hints on the orbital physics in AFM- $E$, we plot in Fig. 3(c) the $\mathrm{YMnO}_{3}$ total charge density of the occupied $e_{g}$ states (in the $[-0.7 ; 0] \mathrm{eV}$ energy range with respect to $E_{F}$ ), which remarkably shows the staggered ordering of the relevant $e_{g}$ orbital occurring in the Mn basal plane.

\section{CONCLUSIONS}

In summary, we have shown via density-functional-based
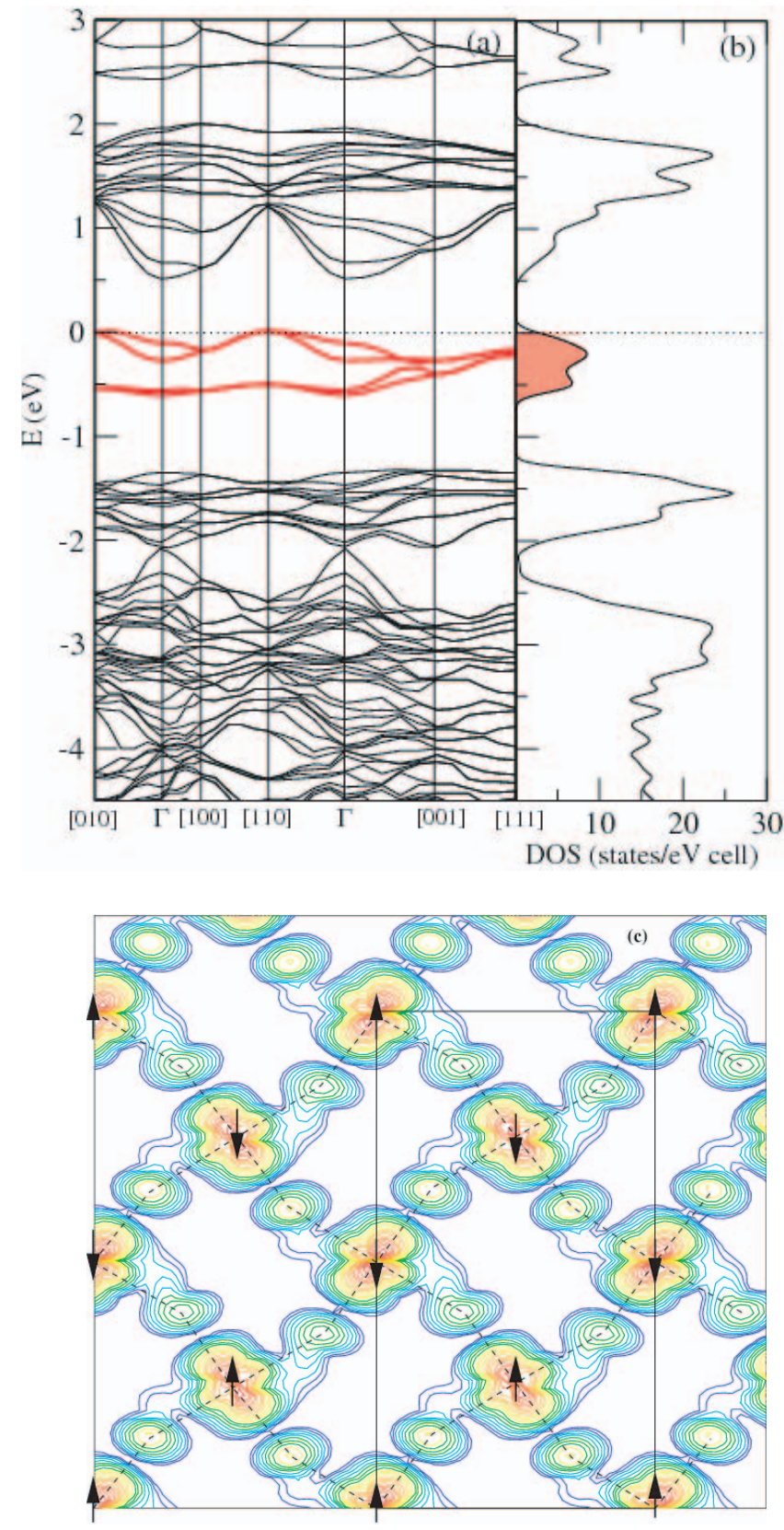

FIG. 3. (Color online) (a) Band structure and (b) total DOS for $\mathrm{YMnO}_{3}$ in the AFM- $E$ magnetic configurations (a Gaussian smearing of $0.1 \mathrm{eV}$ has been used in the DOS calculation). (c) Total charge-density contour plots for occupied $e_{g}$ states. Arrows denote the Mn spins; the solid lines mark the unit cell.

methods that in manganites with highly distorted $\mathrm{MnO}_{6}$ octahedra, such as $\mathrm{HoMnO}_{3}, \mathrm{TbMnO}_{3}$, and $\mathrm{YMnO}_{3}$, the insulating AFM- $E$ phase is stabilized. This is in excellent agreement with experiments, where the AFM- $E$ is observed for $\mathrm{HoMnO}_{3}$. The calculated exchange parameters show that $\mathrm{GdFeO}_{3}$-like distortions-that increase along the rare-earth series-reduce the in-plane nearest-neighbor FM superexchange interaction along the Mn-O-Mn path. This interaction, along with the delicate interplay with the AFM nextnearest-neighbor superexchange along planar Mn-O-O-Mn 
paths, is ultimately responsible for the stabilization of the peculiar AFM- $E$ spin arrangement. Although the approximations made in this study (such as partially inaccurate treatment of correlation effects and atomic relaxations, etc.) should well be tested in the near future, we hope that these results will stimulate more works-both from theory and experiments - to ascertain the role played by the AFM- $E$ magnetic configuration in the manganites framework.
${ }^{1}$ Y. Tokura, Rep. Prog. Phys. 69, 797 (2006), and references therein.

${ }^{2}$ M. B. Salamon and M. Jaime, Rev. Mod. Phys. 73, 583 (2001), and references therein.

${ }^{3}$ T. Kimura, T. Goto, H. Shintani, K. Ishizaka, T. Arima, and Y. Tokura, Nature (London) 426, 55 (2003).

${ }^{4}$ M. Kenzelmann, A. B. Harris, S. Jonas, C. Broholm, J. Schefer, S. B. Kim, C. L. Zhang, S. W. Cheong, O. P. Vajk, and J. W. Lynn, Phys. Rev. Lett. 95, 087206 (2005).

${ }^{5}$ A. Munoz, M. T. Casais, J. A. Alonso, M. J. Martinez-Lope, J. L. Martinez, and M. T. Fernandez-Diaz, Inorg. Chem. 40, 1020 (2001).

${ }^{6}$ J. A. Alonso, J. L. Garcia-Munoz, M. T. Fernandez-Diaz, M. A. G. Aranda, M. J. Martinez-Lope, and M. T. Casais, Phys. Rev. Lett. 82, 3871 (1999); J. L. Garcia-Munoz, J. RodriguezCarvajal, and P. Lacorre, Phys. Rev. B 50, 978 (1994).

${ }^{7}$ E. O. Wollan and W. C. Koehler, Phys. Rev. 100, 545 (1955).

${ }^{8}$ T. Hotta, M. Moraghebi, A. Feiguin, A. Moreo, S. Yunoki, and E. Dagotto, Phys. Rev. Lett. 90, 247203 (2003).

${ }^{9}$ T. Kimura, S. Ishihara, H. Shintani, T. Arima, K. T. Takahashi, K. Ishizaka, and Y. Tokura, Phys. Rev. B 68, 060403 (2003).

${ }^{10}$ E. Wimmer, H. Krakauer, M. Weinert, and A. J. Freeman, Phys. Rev. B 24, 864 (1981).

${ }^{11}$ http://www.flapw.de

${ }^{12}$ L. Nordström and A. Mavromaras, Europhys. Lett. 49, 775
(2000); M. Divis, K. Schwarz, P. Blaha, G. Hilscher, H. Michor, and S. Khmelevskyi, Phys. Rev. B 62, 6774 (2000).

${ }^{13}$ V. Anisimov, F. Aryasetiawan, and A. I. Liechtenstein, J. Phys.: Condens. Matter 9, 767 (1997).

${ }^{14}$ A. B. Shick, A. I. Liechtenstein, and W. E. Pickett, Phys. Rev. B 60, 10763 (1999).

${ }^{15}$ J. A. Alonso, M. J. Martinez-Lope, M. T. Casais, and M. T. Fernandez-Diaz, Inorg. Chem. 39, 917 (2000).

${ }^{16}$ T. Mizokawa, D. I. Khomskii, and G. A. Sawatzky, Phys. Rev. B 60, 7309 (1999).

${ }^{17}$ P. Ravindran, A. Kjekshus, H. Fjellvag, A. Delin, and O. Eriksson, Phys. Rev. B 65, 064445 (2002).

${ }^{18}$ N. Hamada, H. Sawada, and K. Terakura, in Spectroscopy of Mott Insulators and Correlated metals, edited by A. Fujimori and Y. Tokura (Springer, Berlin, 1995); H. Sawada, Y. Morikawa, K. Terakura, and N. Hamada, Phys. Rev. B 56, 12154 (1997).

${ }^{19}$ Incidentally, we note that our results for $\mathrm{LaMnO}_{3}$ are in overall good agreement with previous first-principles calculations, such as Refs. 17 and 21.

${ }^{20}$ Model Hamiltonians for manganites generally include an orbitalordering term in addition to spin-spin coupling, which, however, goes beyond the scope of the present work.

${ }^{21}$ I. Solovyev, N. Hamada, and K. Terakura, Phys. Rev. B 53, 7158 (1996). 